\title{
Safety and efficacy of the Novavax vaccine-a narrative review
}

\author{
Sanny Zi Lung Choo ${ }^{\mathrm{a}}$, Shyh Poh Teo ${ }^{\mathrm{a}, *}$ \\ ${ }^{a}$ Department of Internal Medicine, Raja Isteri Pengiran Anak Saleha (RIPAS) Hospital, Jalan Putera Al-Muhtadee Billah, \\ Bandar Seri Begawan, BA1710, Brunei Darussalam.
}

\begin{abstract}
Mass vaccination programs are a public health priority for managing the global coronavirus disease (COVID-19) pandemic. The NVX-CoV2373 vaccine is being developed by Novavax. It consists of a SARS-CoV-2 spike glycoprotein subunit (NVX-CoV2373), which has been shown to have structural stability with $\mathrm{pH}$ and temperature perturbations, and the saponin-based Matrix-M adjuvant, which is added to enhance the B- and T-cell-mediated immune response. Animal studies in mice, olive baboons, and cynomolgus macaques demonstrated the potential of this vaccine in protecting the respiratory tract against COVID-19. Subsequent phase 1 and 2 trials then confirmed its safety and the dose-sparing potential of Matrix-M. The results led to the use of a low dose $(5 \mu \mathrm{g})$ of NVX-CoV2373 in phase 3 trials. In a phase 3 trial involving 14,039 participants, the vaccine efficacy rate was 89.7\% (prevention of symptomatic infection). Local and systemic adverse events were mild and self-limiting; commonly reported symptoms included injection-site pain and tenderness, headache, myalgia, and fatigue. A subgroup study confirmed the safety and efficacy of co-administering the NVX-CoV2373 vaccine and the seasonal influenza vaccine. Overall, the vaccine has been found to be safe and effective, meeting the minimum vaccine efficacy rate of $50 \%$ to be considered for COVID-19 vaccine emergency use listing approval.
\end{abstract}

Keywords: Clinical trials, COVID-19, COVID-19 vaccine, immunology

\section{Introduction}

The global coronavirus disease (COVID-19) pandemic, caused by severe acute respiratory syndrome coronavirus 2 (SARS-CoV-2), has affected many lives. As of October 13,2021 , there were $238,521,855$ confirmed cases of COVID-19 and 4,863,818 deaths worldwide. Vaccines offer hope for containing this infectious disease, and $6,364,021,792$ vaccine doses had been administered as of October 9, 2021 [1]. Currently, the COVID-19 vaccines that have received the World Health Organization's Emergency Use Listing (EUL) are the Pfizer-BioNTech, Moderna, AstraZeneca, Sinopharm, Sinovac, and Janssen vaccines, which are variously based on mRNA, an adenoviral vector, or inactivated virus [2]. The NVX-CoV2373

\footnotetext{
* Corresponding author: Shyh Poh Teo

Mailing address: Department of Internal Medicine, Raja Isteri Pengiran Anak Saleha (RIPAS) Hospital, Jalan Putera AlMuhtadee Billah, Bandar Seri Begawan, BA1710, Brunei Darussalam.

Email: shyhpoh.teo@moh.gov.bn

Received: 25 October 2021 / Accepted: 07 December 2021
}

vaccine, produced by Novavax, is a protein-based vaccine that has completed a phase 3 trial, and it is hoped that it will be rolled out shortly. This narrative review summarizes the Novavax vaccine trial findings.

\section{Methods}

The PubMed, MEDLINE, SCOPUS, and Google Scholar databases were searched using the search terms 'COVID-19 vaccine,' 'protein subunit vaccine,' 'immunisation,' 'novavax,' and 'NVX-CoV2373' on October 1, 2021. All relevant studies were included in the review.

\section{Development of NVX-CoV2373}

NVX-CoV2373 is a SARS-CoV-2 spike glycoprotein subunit derived from the full-length spike (S) protein. The SARS-CoV-2 S gene that encodes the 1,273 amino acid $S$ protein was mutated at the native furin cleavage site (RRAR to QQAQ) to develop the full-length BV2365 variant, which is protease resistant. Two additional proline substitutions, at positions K986P and V987P, were introduced to provide further stability in the double mutant NVX-CoV2373. These genes were then codon-optimized 
for expression in Sf9 (Spodoptera frugiperda) cells. When purified SARS-CoV-2 (wild-type), BV2365, and NVXCoV2373 proteins were compared in 48-hour tests involving incubation at $\mathrm{pH}$ extremes $(\mathrm{pH} 4$ and $\mathrm{pH} 9)$, prolonged agitation, freeze/thaw cycles, or elevated temperatures $\left(25^{\circ} \mathrm{C}\right.$ and $\left.37^{\circ} \mathrm{C}\right)$. Compared to the other proteins, NVXCoV2373 was found to have significantly greater structural stability, and its human angiotensin I-converting enzyme-2 (hACE2) receptor-binding activity was minimally affected after these stressors [3].

The Novavax vaccine is a combination of NVX-CoV2373 and the saponin-based Matrix-M adjuvant. Subcutaneous administration of Matrix- $\mathrm{M}$ in the absence of an antigen has been shown to promote leukocyte recruitment to the nearby lymph nodes and spleen in mice, with improved activation and maturation of immune cells, especially dendritic cells, to enhance uptake, processing, and presentation of antigens [4]. Supplementing subunit vaccines with Matrix-M has been shown to enhance B- and T-cell immune stimulation in response to the vaccine, with the advantage of dose-sparing potential [5]. The published studies on the development of NVX-CoV2373 are shown in Figure 1.

\section{Preclinical animal studies}

The immunogenicity of NVX-CoV2373 (0.01, 0.1, 1, and $10 \mu \mathrm{g}$ doses) with $5 \mu \mathrm{g}$ Matrix-M as a single dose or in a prime and boost regimen at a 14-day interval was evaluated in mice. This study showed that, in mice, a dose of at least $0.1 \mu \mathrm{g}$ was required to induce anti-S immunoglobulin $\mathrm{G}$ (IgG) titers by day 21 . For all doses tested, a prime and boost regimen induced significantly higher anti-S IgG titers at least seven days after the booster dose compared to before the booster dose. Titers were further enhanced by the addition of Matrix-M ( $v s 10 \mu \mathrm{g}$ NVX-CoV2373), achieving a ten times or higher dose-sparing effect. Across all dose amounts, the prime-boost approach with NVXCoV2373 and Matrix-M induced high titer antibodies that impaired the binding of hACE2 receptors to $\mathrm{S}$, with subsequent neutralization of SARS-CoV-2 cytopathy on Vero E6 cells.

The immunized mice were then administered 105 plaque forming units (pfu)/mouse of SARS-CoV-2 (WA1 strain). While the prime-only mice immunized with NVXCoV2373 and Matrix-M had a dose-dependent response to the viral titer, with no detectable virus at the $10 \mu \mathrm{g}$ dose, the prime/boost immunization with at least $0.1 \mu \mathrm{g}$ NVX-CoV2373 resulted in undetectable lung virus loads. The viral load suppression correlated with protection from weight loss after infection and inflammation in lung his-

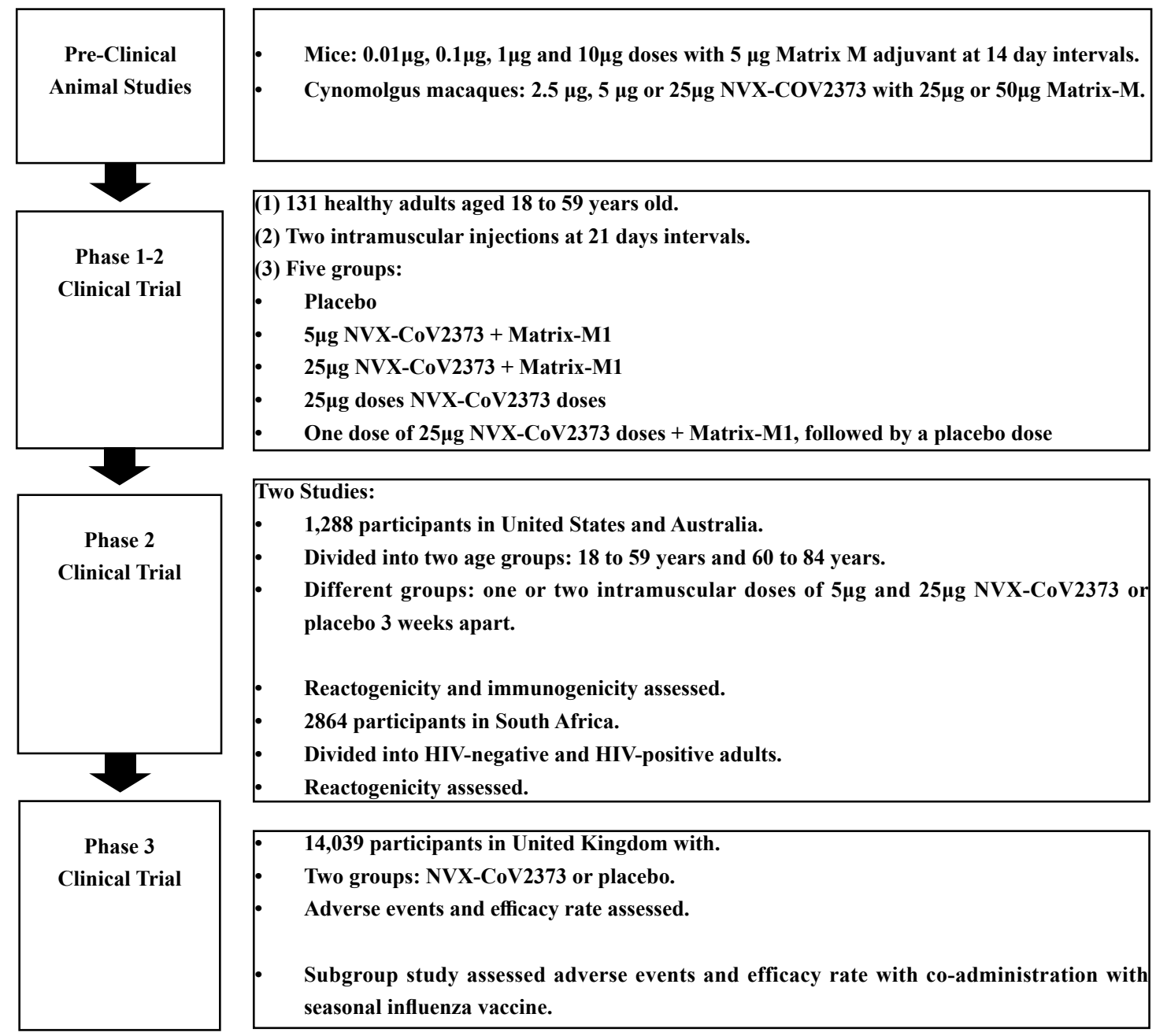

Figure 1. Stages of development of NVX-CoV2373 vaccine. 
topathology. Cell-mediated immune responses were also elicited, with a higher proportion of multi-functioning phenotypes within both cluster of differentiation 4 positive (CD4+) and CD8+ T-cells, with corresponding increased production of tumour necrosis factor- $\alpha$, interferon- $\gamma$, and interleukin- 2 cytokines. These findings in mice were replicated in olive baboons immunized with two doses of 1,5, and $25 \mu \mathrm{g}$ NVX-CoV2373 (plus $50 \mu \mathrm{g}$ Matrix-M) 21 days apart [3].

The vaccine candidate was then tested in a nonhuman primate model: cynomolgus macaques that were at least three years old. Single doses of 2.5, 5, or $25 \mu \mathrm{g}$ NVX-CoV2373 and 25 or $50 \mu \mathrm{g}$ Matrix-M were found to induce anti-S IgG at day 21. Antibody titers increased significantly two weeks after the booster dose, with anti-S IgG titers surpassing those in convalescent human serum by 6.9 to 14.2 times. There were correspondingly similar increases in the hACE2 receptor inhibition titers and SARS-CoV-2 neutralizing antibody geometric mean titers (GMTs). When the macaques were exposed to SARS-CoV-2 (in their lower and upper airways), the placebo recipients were found to have an average of 9,131 single-guide ribonucleic acid (sgRNA) copies/ml in the bronchioalveolar lavage two days post-challenge, as opposed to no detectable sgRNA in the immunized group. The control group animals were found to have moderate to severely inflamed bronchi and alveoli, with minimal inflammation in immunized macaques seven days post-challenge. This demonstrates the ability of NVX-CoV2373 to prevent viral replication in the upper respiratory tract and pulmonary disease in the lower respiratory tract, which could be tested in human trials [6].

\section{Phase 1-2 clinical trial}

A phase 1-2 trial was conducted, for which 131 healthy adults aged 18-59 years were recruited. They were administered two intramuscular injections at 21-day intervals. The participants were randomly assigned into five groups: placebo doses; $5 \mu \mathrm{g}$ NVX-CoV2373 with MatrixM1; $25 \mu \mathrm{g}$ NVX-CoV2373 with Matrix-M1; $25 \mu \mathrm{g}$ NVXCoV2373 without Matrix-M1; and one dose of $25 \mu \mathrm{g}$ NVX-CoV2373 with Matrix-M1, followed by a placebo dose. Matrix-M and Matrix-M1 are almost identical adjuvants and are manufactured by the same company. The only difference is the ratio of Matrix A and Matrix $\mathrm{C}$ used to constitute the adjuvants; where Matrix-M and MatrixM1 using a ratio of Matrix A: Matrix C of 91:9 and 85:15 respectively. This trial was conducted at two Australian study sites (Nucleus Network, Herston, Queensland, and Melbourne, Victoria). In terms of reactogenicity, the majority of participants had absent or mild local and systemic side effects after the first and second doses in all five groups. Eight participants (at most two in each subgroup) experienced severe systemic events after dose two. The most common were joint pain and fatigue, which were self-limiting.

In terms of immunogenicity, enzyme-linked immunosor- bent assay (ELISA) results showed that the anti-S IgG geometric mean fold rises in all the adjuvanted regimens exceeded those in the non-adjuvanted regimens by 10 times. This further increased by a factor of 8 a week after the second dose, doubling again by 14 days and exceeding levels found in the convalescent serum of hospitalized patients with COVID-19. The immunogenicity resulting from two doses of the 5 and $25 \mu \mathrm{g}$ vaccines with adjuvant was similar, confirming the dose-sparing capability of Matrix-M1. The adjuvanted regimens also induced effective polyfunctional CD4+ T-cell responses and associated TNF- $\alpha$, IFN- $\gamma$, and IL-2 production when cells were stimulated by $\mathrm{S}$ protein. The Th1-type response was preferred over the Th2-type response, and there was minimal IL-5 and IL-13 cytokine production [7].

\section{Phase 2 clinical trials}

After the safety and immunogenicity data were confirmed for up to two weeks after the second dose (day 35), the next stage of the phase 2 trial was a randomized study. This was conducted between August 24 and September 25,2020 , and involved 1,288 randomized participants at 17 sites in the United States and Australia. There were two groups based on age: 18-59 years and 60-84 years. NVXCoV2373 doses were all adjuvanted with Matrix-M1. Participants received either one or two intramuscular doses of 5 and $25 \mu \mathrm{g}$ NVX-CoV2373 or placebo three weeks apart. Regardless of age, solicited local adverse events were more common in the participants who received NVXCoV2373 compared to placebo; they were mostly selflimiting tenderness (up to $59 \%$ ) and pain (up to $38 \%$ ). Adverse events were more likely in younger participants, with the higher dose, and after dose two. In terms of systemic symptoms, muscle pain was the most frequent symptom $(20 \%)$. This was also observed in the placebo group after one dose, in which the symptoms were mainly grade 1 and short lasting. Fever occurred in less than $2 \%$ of the vaccine recipients. After dose two, the most common systemic symptoms were fatigue $(43 \%)$, muscle pain $(41 \%)$, headache $(34 \%)$, and malaise $(30 \%)$; again, these were low-grade, self-limiting symptoms regardless of age. Both doses of NVX-CoV2373 induced anti-S IgG GMTs and neutralizing antibodies, exceeding the levels found in convalescent sera of outpatients and hospitalized patients with COVID-19. Overall, this demonstrated that the administration of NVX-CoV2373 induced high immunogenicity and that it was well tolerated by younger and older people. Thus, $5 \mu \mathrm{g}$ NVX-CoV2373 progressed to phase $2 \mathrm{a} / \mathrm{b}$ and phase 3 studies [8].

A separate phase 2 trial was conducted in South Africa between August 17 and November 25, 2020, with 2,864 randomized seronegative participants at 16 sites. There were two groups: human immunodeficiency virus (HIV)negative participants 18-84 years old and HIV-positive participants 18-64 years old who were medically well. Participants received either two doses of $5 \mu \mathrm{g}$ NVXCoV2373 or placebo three weeks apart. 
In both groups, solicited local adverse events were more common in those who received NVX-CoV2373 compared to placebo, without any significant difference in incidence after the first or second dose. The duration of local adverse events was slightly longer after dose two but was within three days. Severe local adverse events were uncommon but were reported more after dose two in vaccine recipients compared to placebo recipients (4\% vs $1 \%$ ). Similarly, reported systemic symptoms were more common in those who received NVX-CoV2373 compared to placebo. Headache, muscle pain, and fatigue were the most frequent symptoms, lasting slightly longer after dose two.

No differences in reactogenicity were found between the HIV-negative and HIV-positive groups, although the latter sample size was small. Symptomatic COVID-19 infections occurred in 15 participants who were vaccinated and in 29 participants who received placebo, corresponding to a vaccine efficacy of $49.4 \%$. The B.1.351 variant was confirmed in $38(93 \%)$ of the 41 samples sent for wholegenome sequencing. Post hoc analysis identified a vaccine efficacy of $43.0 \%$ against the B.1.351 variant [9].

\section{Phase 3 clinical trial}

A phase 3 trial was conducted between September 28 and November 29, 2020, across 33 locations in the United Kingdom, with 14,039 randomized participants aged between 18 and 84 years. Among the participants, 27.9\% were aged 65 years or older, while $44.6 \%$ had coexisting illnesses.

Similar to the phase 2 trials, local adverse events occurred more frequently in the vaccine group compared to the placebo group, and were more commonly reported after the second dose compared to the first $(79.6 \%$ vs $57.6 \%)$. The most frequent local adverse events were injection-site pain and tenderness, which were mostly mild to moderately severe and short-lived ( $<3$ days). There were more reported local adverse events in the younger participants than in the older participants (65 years and above). The reported systemic adverse events were higher in the vaccine group than in the placebo group after dose one $(45.7 \%$ vs $36.3 \%)$ and dose two (64.0\% vs $30.0 \%)$. The most frequently solicited systemic symptoms were headache, muscle pain, and fatigue, which were mostly grade 1 or 2 and lasted less than two days. Systemic adverse events were more commonly reported in younger vaccine recipients, with grade 4 systemic adverse events reported in three participants from the vaccine group. Two participants reported grade 4 fever $\left(>40{ }^{\circ} \mathrm{C}\right)$ : one after dose one and the other after dose two. Myocarditis was diagnosed in a vaccinated participant three days after dose two, and they fully recovered after two days of hospitalization [10].

Of the 14,039 participants, 10 had symptomatic COVID-19 at least one week after dose two of the vaccine compared to 96 who received placebo, equating to an efficacy rate of $89.7 \%$. The 10 participants from the vaccine group were all aged 65 years and older. Five participants had severe COVID-19, all of whom received placebo. There were two COVID-19-related deaths in this study, one in the vaccine group and one in the placebo group. The participant in the vaccine group who died developed COVID-19 symptoms a week after the first dose and passed away eight days later [10].

There were several limitations associated with this trial. The study was performed in the United Kingdom only, and the majority (94.5\%) of the participants were White. Thus, it is unclear whether the efficacy findings can be extrapolated to other ethnic groups. The study also excluded pregnant women, those aged under 18 years, people receiving immunosuppressants, and those with immunodeficiency. Thus, further studies are required to assess the safety and efficacy of NVX-CoV2373 in these groups. The trial was also conducted when the B.1.351 (Alpha) variant was prevalent; however, data on the efficacy of this vaccine against the B.1.617.2 (Delta) variant, which is currently causing outbreaks in many parts of the world, would be more relevant.

A subgroup study of this phase 3 trial was performed with the first 400 participants who met the inclusion criteria without contraindications to the influenza vaccine. After randomization to receive either NVX-CoV2373 or placebo, the participants received an open-label influenza vaccine with their first dose. Compared to the main study, the subgroup participants were younger and had fewer comorbidities. Local reactions were more prevalent in the co-vaccinated group (70.1\%) than in the NVX-CoV2373 alone group (57.6\%) and in the influenza vaccine (coadministered with placebo) group (39.4\%). Systemic reactions were also more common in those co-vaccinated (60.1\%) than in the NVX-CoV2373 alone group (45.7\%). Commonly reported systemic reactions were myalgia, fatigue, and fever, which were mostly mild and self-limiting. The likelihood of severe systemic reactions was increased more than twofold in the co-vaccinated group compared to the NVX-CoV2373 alone group (2.9\% vs $1.3 \%$ ). However, there were no differences in the frequency of all adverse events between the co-vaccinated group and the NVX-CoV2373 alone group (18.4\% vs 17.6\%).

More importantly, the immunogenicity of NVX-CoV2373 was not affected by co-administration with the seasonal influenza vaccine ( $87.5 \%$ vs $89.7 \%$ in the main study). This supports the potential co-administration of COVID-19 and seasonal influenza vaccines, particularly for at-risk groups, such as older people, pregnant people, and those with multiple comorbidities [11].

\section{Conclusion}

The NVX-CoV2373 vaccine phase 3 trial demonstrated its safety and efficacy. The vaccine met the minimum vaccine efficacy rate of $50 \%$ to be considered for COVID-19 vaccine EUL approval. Adverse events were mild and selflimiting, occurring more frequently in younger people and after the second dose. Co-administration of this vaccine with the seasonal influenza vaccine is a practical approach 
for reaching population groups at high risk of developing complications from viral respiratory infections.

\section{Declarations}

Authors' contributions: Choo SZL and Teo SP were involved in conception of the work, acquisition, analysis and interpretation of data, as well as drafting and finalizing the manuscript.

Availability of data and materials: Not applicable.

Financial support and sponsorship: Not applicable.

Conflicts of interest: All authors declare that there are no conflicts of interest.

Ethical approval and consent to participate: Not applicable.

\section{References}

1. WHO. WHO Coronavirus (COVID-19) Dashboard. 2021.

2. WHO. Status of COVID-19 vaccines within WHO EUL/PQ evaluation process. 2021.

3. Tian J-H, Patel N, Haupt R, et al. SARS-CoV-2 spike glycoprotein vaccine candidate NVX-CoV2373 immunogenicity in baboons and protection in mice. Nature Communications, 2021, 12(1): 1-14.

4. Reimer J M, Karlsson K H, Lövgren-Bengtsson K, et al.
Matrix- $\mathrm{M}^{\mathrm{TM}}$ adjuvant induces local recruitment, activation and maturation of central immune cells in absence of antigen. PloS one, 2012, 7(7): e41451.

5. Bengtsson K L, Song H, Stertman L, et al. Matrix-M adjuvant enhances antibody, cellular and protective immune responses of a Zaire Ebola/Makona virus glycoprotein (GP) nanoparticle vaccine in mice. Vaccine, 2016, 34(16): 1927-35.

6. Guebre-Xabier M, Patel N, Tian J-H, et al. NVX-CoV2373 vaccine protects cynomolgus macaque upper and lower airways against SARS-CoV-2 challenge. Vaccine, 2020, 38(50): 7892-6.

7. Keech C, Albert G, Cho I, et al. Phase 1-2 trial of a SARSCoV-2 recombinant spike protein nanoparticle vaccine. New England Journal of Medicine, 2020, 383(24): 232032.

8. Formica N, Mallory R, Albert G, et al. Evaluation of a SARS-CoV-2 Vaccine NVX-CoV2373 in Younger and Older Adults. medRxiv, 2021.

9. Shinde V, Bhikha S, Hoosain Z, et al. Efficacy of NVXCoV2373 Covid-19 Vaccine against the B. 1.351 Variant. New England Journal of Medicine, 2021, 384(20): 1899909.

10. Heath P T, Galiza E P, Baxter D N, et al. Safety and efficacy of NVX-CoV2373 Covid-19 vaccine. New England Journal of Medicine, 2021, 385(13): 1172-83.

11. Toback S, Galiza E, Cosgrove C, et al. Safety, Immunogenicity, and Efficacy of a COVID-19 Vaccine (NVXCoV2373) Co-administered With Seasonal Influenza Vaccines. medRxiv, 2021.

Cite this article as: Choo S Z L, Teo S P. Safety and efficacy of the Novavax vaccine-a narrative review[J]. Aging Pathobiology and Therapeutics, 2021, 3(4): 102-106. 